\title{
¿Cachái? y sus equivalentes funcionales en el habla santiaguina: análisis pragmático y sociolingüístico de los marcadores interrogativos de control de contacto*
}

\author{
Eileen Lewis Vergara** \\ Abelardo San Martín Núñez ${ }^{* * *}$
}

\section{Resumen}

En este artículo se analizan el comportamiento pragmático y la estratificación sociolingüística de los "marcadores interrogativos de control de contacto" en el habla santiaguina. En el estudio se abordan los subvalores de dichos marcadores de función predominantemente fática, en relación con el tipo de unidad discursiva y su posición preferente, así como la correlación de su empleo con factores sociodemográficos de los hablantes. Los resultados muestran que, a pesar de haberse registrado un repertorio amplio de partículas, el marcador interrogativo de control de contacto más frecuente es ¿cachái? Igualmente, el factor sociodemográfico más sensible a su empleo fue la edad de los sujetos.

Palabras clave: ¿Cachái?, función fática, marcadores del discurso, pragmática, sociolingüística, español de Chile.

\section{¿Cachái? and their functional equivalents in the Santiago, Chile speech: pragmatic and sociolinguistic analysis of interrogative contact control markers}

\begin{abstract}
This article analyzes the pragmatic behavior and sociolinguistic stratification of the "interrogative contact control markers" in the Santiago, Chile speech. The study approaches the sub-values of these predominantly phatic function markers, in relation to the type of discursive u nit and its preferential position, as well as the correlation of its use with sociodemographic factors of the speakers. Although a broad repertoire of particles was recorded, the results show that the most frequent contact control interrogative marker
\end{abstract}

Este estudio forma parte del proyecto FONDECYT Regular 1161422, "Los marcadores del discurso en el español hablado en Santiago de Chile: análisis pragmático y sociolingüístico”. Se reformulan aquí los principales hallazgos de la tesis de licenciatura de Eileen Lewis, investigación dirigida por Abelardo San Martín.

Chilena. Licenciada en Lengua y Literatura Hispánica Mención Lingüística de la Universidad de Chile.Santiago,Chile.eileen.lewis@ug.uchile.cl

*** Chileno. Doctor en Filología Hispánica de la Universidad de Valladolid. Académico de la Universidad de Chile, Santiago, Chile. asmartin@uchile.cl 
found is ¿cachái? Likewise, the socio-demographic factor most sensitive to its use was the age of the subjects.

Keywords: ¿cachái?, phatic function, discourse marker, pragmatics, sociolinguistics, Chilean Spanish.

\section{Introducción}

La necesidad de controlar el contacto con nuestro interlocutor y de comprobar su comprensión durante el intercambio constituye uno de los requerimientos básicos para el funcionamiento de una conversación, por lo que la función fática fue tempranamente identificada en los estudios del lenguaje y la comunicación (Malinowski, 1984[1923]; Jakobson, 1975). Como señala Cestero (2002, p. 618), la función fática es predominante cuando el hablante emplea recursos verbales y no verbales "con el fin de establecer, prolongar o interrumpir la comunicación, asegurase de que el canal de comunicación está abierto y funciona, llamar la atención del interlocutor o confirmar si su atención se mantiene". Para ello, según la misma autora, los hablantes cuentan con recursos de diverso tipo, entre los que destacan los marcadores del discurso, que pueden asumir una forma interrogativa comprobativa (ime entiendes?, ¿verdad?), ordenadora del discurso (bueno, claro) o imperativa de carácter vocativo (oye, mira). En el presente trabajo nos ocuparemos de los marcadores del primer tipo, a los que también se les ha llamado "apéndices comprobativos" (Ortega, 1985), si bien nosotros preferiremos el término "marcadores interrogativos del control de contacto" (San Martín, 2011). El objetivo general de este artículo es analizar el comportamiento pragmático y la distribución social del empleo de dichos marcadores en una muestra socialmente estratificada de 120 entrevistas realizadas a hablantes santiaguinos. Nuestros objetivos específicos son: a) identificar los marcadores interrogativos que cumplen la función de control de contacto en la muestra; b) determinar el comportamiento pragmático de dichos marcadores, en términos de sus subvalores, los tipos de unidades discursivas en los que operan y su posición preferente dentro de estas unidades y c) correlacionar sus frecuencias de uso con los factores sociodemográficos: sexo-género, edad y grupo socioeconómico de los informantes. Por otro lado, las hipótesis que someteremos a comprobación son: a) 
si bien existe un paradigma amplio de marcadores interrogativos de control de contacto, ¿cachái? es el más frecuente; b) dichos marcadores son polifuncionales en cuanto a sus subvalores, aunque comparten una función fática nuclear y c) los factores sociodemográficos de los sujetos inciden de manera irregular en su empleo. La naturaleza del presente estudio es cuantitativa y correlacional y se enmarca en las directrices metodológicas y teóricas aplicadas por San Martín (2011, 2013, 2015, 2016a y 2016b) para el estudio variacionista de los marcadores del discurso en el español de Chile, en la que se combinan estrategias analíticas de la pragmática y la sociolingüística ${ }^{1}$.

\section{Marco teórico}

En este estudio abordamos un tipo específico de marcadores del discurso que, en términos generales, Martín Zorraquino y Portolés (1999) llaman enfocadores de la alteridad y que Portolés (2001) denomina, siguiendo a Briz (2001), Marcadores de control de contacto, los que, de acuerdo con este último, se refieren a partículas de tipo metadiscursivo. A este grupo pertenecen tanto marcadores de forma interrogativa (por ejemplo, ino?, ¿eh?, ¿ah?, ¿sí?, ¿entiendes?, ¿verdad?, ¿sabes?) como de forma afirmativa (por ejemplo, oye, escucha, mira, fíjate). Nos ocuparemos aquí, específicamente, de los marcadores de contacto con forma interrogativa que, funcionalmente, desde el punto de vista pragmático-discursivo, mantienen el contacto y controlan la comprensión del interlocutor, por lo tanto, cumplen la "función fática" del lenguaje (Malinowski, 1984[1923]; Jakobson, 1975), más específicamente, el "mantenimiento de la atención interlocutiva" (Casado Velarde, 1998, p. 65). Nuestro concepto de "marcador interrogativo de control de contacto", por lo tanto, se restringe a aquellas partículas periféricas con forma interrogativa y de valor metadiscursivo, mediante las cuales los hablantes mantienen la atención y controlan la comprensión del resto de los participantes en un intercambio comunicativo. Este tipo específico de unidades en español se ha estudiado en el marco de obras de carácter general. Fuentes, por ejemplo, estudia la función fática (1990a) y la función apelativa (1990b) de algunos operadores en el español de Sevilla desde el punto de vista sociolingüístico.

Una panorámica crítica del estudio de la variación dialectal y sociolingüística de los marcadores del discurso se encuentra en Carbonero y Santana (2010). 
Asimismo, se los ha analizado en términos de "apéndices comprobativos o justificativos" (Ortega, 1985 y 1986) o, recurriendo a su denominación en inglés, como "preguntas confirmatorias" (tag questions) (Móccero, 2010), resaltando su valor como apéndices mediante los cuales los hablantes intentan comprobar o confirmar la aceptación de sus propias afirmaciones u opiniones por parte de los interlocutores. Aunque nuestro objeto de análisis primordial es la función fática de estos marcadores, también resulta de gran interés su polivalencia funcional ${ }^{2}$.

Según Cestero (2002 y 2003), los marcadores interrogativos de control de contacto cumplen una función fática externa de la que se desprende una función fática interna (Pons, 1998), en la que la llamada de atención al interlocutor destaca un elemento del discurso como más relevante frente a otros en términos de jerarquía y organización, de modo que involucra la actitud del hablante frente a su discurso. Por lo tanto, consiste en una función modalizadora que establece una relación entre el hablante y el contenido proposicional de su discurso (modalización epistémica), o bien, que refiere a la actitud valorativa del hablante frente a su discurso (modalización deóntica). De acuerdo con Cestero (2002, p. 231), "los dos tipos de función fática van unidos, de manera que al utilizar un apéndice apelativo, la llamada siempre es doble: hacia el interlocutor y hacia el discurso"; por consiguiente, se trata de partículas polifuncionales. Su forma interrogativa determina que se les otorgue con mucha frecuencia un valor comprobativo, en términos de partículas de petición de confirmación, que constituyen una manera de proyectar un acuerdo con el interlocutor o una manera de pedir su opinión (Móccero, 2010). Cuando requiere respuesta, se trata de una pregunta plena y, frecuentemente, se posiciona al final del enunciado operando de modo secundario como un marcador de cierre. Cuando no la requiere, resulta ser efectivamente una "pregunta confirmatoria", es decir, "aquella pregunta destinada a corroborar información u opinión, ya sea producida por el hablante acerca de su propia contribución [...] o producida por el interlocutor para confirmar si oyó/entendió bien” (Móccero, 2010, p.

\footnotetext{
2 Rodríguez (2009), en particular, distingue las funciones pragmáticas de $i n o ? y_{i} e h$ ?, destacando el funcionamiento fático de dichas partículas, más allá de su mero valor comprobativo. Móccero (2010), además, estudia el valor de las partículas ¿no? $y_{i} s i$ ? como marcadores de posicionamiento intersubjetivo en una muestra de conversaciones en español de Argentina. Asimismo, Ramírez Gelbes (2003) estudia el contenido procedimental de la partícula $e h$, también con base en muestras del español argentino. Por su parte, García Vizcaíno (2005) investiga las diferencias entre los apéndices moralizadores ¿no? y ¿eh? en el español peninsular a la luz de los estudios de la cortesía verbal.
} 
70-71). Por último, debido a que en la mayoría de los casos se trata de partículas fáticas y apelativas, García Vizcaíno (2005) y Landone (2009) las vinculan estrechamente con las máximas de cortesía.

En cuanto al estudio de este tipo de marcadores del discurso en el español de Chile, Rabanales y Contreras (1995) estudian las funciones de las muletillas en el habla culta de Santiago de Chile, en general, entre las cuales incluyen las de función fática (oye, mira, ¿no es cierto?, ¿no?, ¿ah?, ¿mm? y ¿ves tú?). Además de proporcionar una clasificación de las funciones de las muletillas en una muestra de diálogos informales de hablantes cultos, entregan datos cuantitativos sobre su empleo, según las variables edad y sexo, resultando ser los jóvenes y los hombres los que más las emplean. En San Martín (2011) abordamos los marcadores interrogativos de control de contacto desde el punto de vista sociolingüístico en una muestra de 54 entrevistas del corpus PRESEEA de Santiago. Aunque se relevaron 12 marcadores que cumplían la misma función, se verificó una abrumadora preponderancia de ¿cachái?, en términos de frecuencia de empleo; marcador muy extendido entre los jóvenes de la muestra, en especial, si bien no de modo exclusivo, en los hombres. Por otra parte, Gille (2015a y b) estudia las funciones pragmáticas de ¿cachái?, considerándolo un apéndice conversacional, en intercambios de estudiantes universitarios chilenos ${ }^{3}$, en los que distingue las siguientes funciones: llamada de atención (función nuclear), gestión intersubjetiva, organización discursiva, autorregulación, organización del discurso y distribución de roles conversacionales. Por último, Mondaca et al. (2014) analizan el comportamiento de ¿cachái? en un corpus de entrevistas sociolingüísticas realizadas a hablantes chilenos de diferente sexo y origen geográfico, correspondientes a cuatro zonas dialectales (norte, centro, sur y sur-austral). Las autoras identifican que la función base de ¿cachái? es captar la atención del interlocutor, de la cual se desprenden otras: solicitud de aprobación, herramienta argumentativa, organización del discurso y autorregulación. Según Mondaca et al. (2014), ¿cachái? es empleado sin diferencias notables por hombres y mujeres en todo Chile, aunque los hombres y los hablantes de la zona central,

Gille (2015a y b) también indaga el origen de ¿ cachái? utilizando fuentes históricas, como el Corpus Diacrónico del Español (CORDE), lexicográficas y literarias, bajo el supuesto de que existe un vínculo entre sus usos históricos y los actuales. Con respecto a su origen, el autor desestima que ¿cachái? sea un préstamo del inglés catch, que constituye la explicación más extendida (cf. Real Academia Española 2001 , s. v. cachar); en cambio, se decanta por considerarlo una derivación del español catar (catar > catear $>$ catiar $>$ cachar), la que ya había propuesto Lenz (1905). 
representada por Valparaíso, superan levemente a las mujeres y a los sujetos de otras zonas del país, respectivamente.

\section{Metodología}

\subsection{Corpus}

El corpus que sirvió de base para nuestro estudio corresponde a 120 entrevistas sociolingüísticas pertenecientes al corpus del grupo de Estudio Sociolingüístico del Español de Chile (ESECH). Las entrevistas fueron realizadas a hablantes santiaguinos, entre 2005 y 2011, por los estudiantes en la asignatura de Sociolingüística de los programas de Licenciatura en Lengua y Literatura Hispánicas y Licenciatura en Lengua y Literatura Inglesas de la Universidad de Chile. En la situación de entrevista, los entrevistadores debían tratar de superar la "paradoja del observador" consiguiendo, de esta forma, una muestra significativa de discurso natural grabado (estilo vernáculo) de hablantes representativos de la comunidad de habla en estudio (Labov, 1983). La transcripción de las entrevistas antes señaladas fue exhaustivamente revisada, sin exclusión de ninguna de las secciones del instrumento.

\subsection{Población y muestra}

En nuestra investigación se consideró la población constituida por hombres y mujeres de la Región Metropolitana de más de 20 años de edad. El cuestionario se aplicó a una muestra por cuotas con afijación uniforme, en la que se divide a la población en estratos o categorías y se asigna una cuota a cada uno de los distintos estratos. La muestra, así conformada, comprende un total de 120 entrevistas realizadas a igual número de sujetos.

\subsection{Procedimiento de estratificación social utilizado en ESECH}

Para la estratificación de los sujetos que conforman la muestra del estudio, se empleó el sistema de adscripción de estatus social empleado en ESECH que se basa en la asignación del puntaje -que se indica entre paréntesis- a los informantes, de acuerdo con las siguientes variables: a) nivel educacional (3), b) profesión u ocupación (2) y c) comuna de residencia (1). La explicación en detalle de dichas variables se encuentra 
en San Martín y Guerrero (2015), que consiste en un procedimiento de estratificación basado en determinados estudios acerca de la realidad socioeconómica chilena. A partir de la asignación de los mencionados puntajes, se definieron cuatro grupos socioeconómicos, definidos según los rangos siguientes: a) Medio alto (MA): 42 - 36, b) Medio (M): 35 - 27, c) Medio bajo (MB): 26 - 18 y d) Bajo (B): $17-6^{4}$.

\subsection{Procedimiento analítico}

Nuestro análisis de los marcadores interrogativos de control de contacto consideró la determinación de sus respectivas frecuencias de ocurrencia en las entrevistas que conforman la muestra. Asimismo, de modo coherente con nuestros objetivos, se observó la incidencia de los siguientes factores sociodemográficos de los hablantes: sexo-género, edad y grupo socioeconómico. El análisis estadístico atendió a dos niveles de observación: a) en términos descriptivos, según frecuencias absolutas y porcentajes de frecuencia y b) en términos interpretativos o inferenciales, con base en la comparación entre las medias o tendencias centrales de los marcadores más frecuentes, esto es, de aquellos con más de 25 ocurrencias ${ }^{5}$.

\section{Análisis de resultados}

\subsection{Análisis pragmático}

Se registraron 2312 casos de la función interrogativa de control de contacto mediante marcadores en la muestra. El paradigma de marcadores que desempeñan dicha función y sus frecuencias en el corpus analizado se presentan en la siguiente tabla:

\footnotetext{
4 El intervalo de cada rango responde a la mayor coincidencia con los puntajes que han establecido los estudios sociológicos y de mercado para la clasificación de estratos socioeconómicos, aunque con las debidas adaptaciones, puesto que en esta muestra no se incluyen ni el grupo de la extrema pobreza ni el de la extrema riqueza.

5 Por lo que se refiere a la descripción de los procedimientos estadísticos aplicados, en este trabajo seguimos las sugerencias e indicaciones de Hernández Campoy y Almeida (2005). El paquete estadístico al que hemos recurrido para la estadística inferencial es el SPSS (Statistical Package for the Social Sciences), versión 15.0 para Windows, específicamente, la prueba Análisis de varianza ANOVA. En atención a que la distribución de los datos, en algunos casos, pudiera ser anormal, se complementará dicho análisis con su análogo de tipo no paramétrico, esto es, Anova de Kruskal Wallis. En ambos casos, el grado de significación se definirá en el $5 \%$, según el cual $\mathrm{p}=<0,05$ será estadísticamente significativo.
} 
Tabla 1. Frecuencia absoluta y porcentaje de los marcadores interrogativos de control de contacto relevados.

\begin{tabular}{|c|c|c|}
\hline Marcador & Frecuencia absoluta & Porcentaje \\
\hline ¿cachái? & 1499 & $64,84 \%$ \\
\hline ¿ya? & 127 & $5,49 \%$ \\
\hline ¿ah? & 104 & $4,50 \%$ \\
\hline ¿te fijah? & 84 & $3,63 \%$ \\
\hline ¿no cierto? & 71 & $3,07 \%$ \\
\hline ¿cierto? & 66 & $2,85 \%$ \\
\hline ¿te fijái? & 62 & $2,68 \%$ \\
\hline ¿me entendíh? & 53 & $2,29 \%$ \\
\hline ¿no? & 47 & $2,03 \%$ \\
\hline ¿no eh cierto? & 38 & $1,64 \%$ \\
\hline ¿mmm? & 27 & $1,17 \%$ \\
\hline ¿me entiendeh? & 19 & $0,82 \%$ \\
\hline ¿sí? & 17 & $0,74 \%$ \\
\hline ¿me entendíh tú? & 16 & $0,69 \%$ \\
\hline ¿entiendeh? & 13 & $0,56 \%$ \\
\hline ¿o no? & 12 & $0,52 \%$ \\
\hline ¿cachái o no? & 10 & $0,43 \%$ \\
\hline ¿vihte? & 8 & $0,35 \%$ \\
\hline ¿te fijah tú? & 7 & $0,30 \%$ \\
\hline ¿entendíh? & 6 & $0,26 \%$ \\
\hline ¿me entiendeh tú? & 5 & $0,22 \%$ \\
\hline ¿me entiende? & 4 & $0,17 \%$ \\
\hline ¿veh tú? & 4 & $0,17 \%$ \\
\hline ¿eh? & 3 & $0,13 \%$ \\
\hline ¿entiendeh tú? & 2 & $0,09 \%$ \\
\hline ¿veh? & 2 & $0,09 \%$ \\
\hline ¿no eh verdad? & 2 & $0,09 \%$ \\
\hline ¿sí o no? & 2 & $0,09 \%$ \\
\hline ¿me cachái? & 1 & $0,04 \%$ \\
\hline ¿de verdad? & 1 & $0,04 \%$ \\
\hline Total & 2312 & $100 \%$ \\
\hline
\end{tabular}

La función principal (o nuclear) de estos marcadores interrogativos es el control de contacto o la función fática externa, es decir, una "función estructural que involucra a hablante e interlocutor, de forma activa, en el intercambio comunicativo" (Cestero, 2002, p.234). Consiste en una manera de llamar la atención del oyente para mantener la comunicación, centrándose tanto en el control sobre la recepción del discurso como 
en la apelación activa al interlocutor. No obstante, algunos autores coinciden en que pueden desempeñar otros valores en la conversación, como estructuradores de la información, modalizadores o partículas comprobativas, que intentaremos sistematizar en lo que sigue, con base en el análisis pragmático de los marcadores relevados en el corpus. A partir de esta función nuclear, por lo tanto, se desprenden las subfunciones de petición de confirmación (marcadores interactivos) y de estructuración de la información (marcadores textuales) 6 . A su vez, de la primera se derivan los valores de modalización deóntica y epistémica y, de la segunda, las funciones de relleno y de cierre de unidad discursiva. A propósito de los valores modalizadores de estos marcadores, cabe hacer algunas precisiones. En primer lugar, su modalización deóntica guarda relación con la petición de confirmación de una opinión o aseveración del hablante hacia el interlocutor, con o sin respuesta requerida, la que puede reemplazarse por una pregunta como "¿no crees u opinas lo mismo?" o “¿no te parece?” En este sentido, según Móccero (2010), constituyen "preguntas confirmatorias" de naturaleza fática, que operan en la esfera de la relación hablante-mensaje, si se intenta corroborar una opinión del interlocutor, y en la esfera de la relación hablante-oyente, si lo que se confirma es un juicio de valor. En segundo término, su modalización epistémica se vincula con la confirmación del contenido proposicional del enunciadodel hablante, ya sea como estrategia de cortesía o bien porque el hablante requiere deuna corroboración del interlocutor, con o sin respuesta requerida ${ }^{7}$, equivalente a preguntas del tipo " $i$ es cierto lo que digo?" o “ ¿no es verdad lo que digo?”; incluso, pueden darse casos en que estas preguntas estén más motivadas semánticamente. Por último, ambas funciones de petición de confirmación se relacionan estrechamente con valores pragmáticos de cortesía, en la medida en que conforman estrategias discursivas de protección y refuerzo de la imagen positiva y negativa del interlocutor. Desde esta última perspectiva, Landone (2009) señala que los marcadores que se vinculan a la modalidad y la relación entre los hablantes y sus interlocutores se relacionan con la cortesía, debido a que pueden manifestar funciones de negociación entre posturas (acuerdo/desacuerdo/ pseudoacuerdo), además de la "modulación de intensidad y de proximidad (para reforzar o buscar la sintonización empática con el interlocutor) y de

La distinción entre marcadores interactivos y textuales la debemos a Cortés y Camacho (2005).

Para Clark y Brennan (1991) el segundo caso, esto es, sin solicitud de respuesta, correspondería a una estrategia de grounding o construcción de un terreno común en la comunicación. 
especificidad (enfocar/desenfocar al interlocutor)"(Landone, 2009, p. 236). Así, configuramos un cuadro general de las funciones de modalización (hablante-mensaje) y de petición de confirmación (hablante-oyente) que se da en una suerte de gradiente o continuum en la interacción verbal: a mayor función de modalización, es decir, a mayor relación del hablante con su mensaje, menores la función de petición de la información, o bien, menor la relación entre el hablante y su interlocutor ${ }^{8}$. Lo que puede expresarse también en términos de cortesía: a mayor modalización (propiamente tal), menor es la búsqueda de acuerdo y, por tanto, menor es la cortesía y viceversa (ver figura 1).

Figura 1. Valores generales derivados de la función de control de contacto mediante marcadores interrogativos en relación con la cortesía verbal.

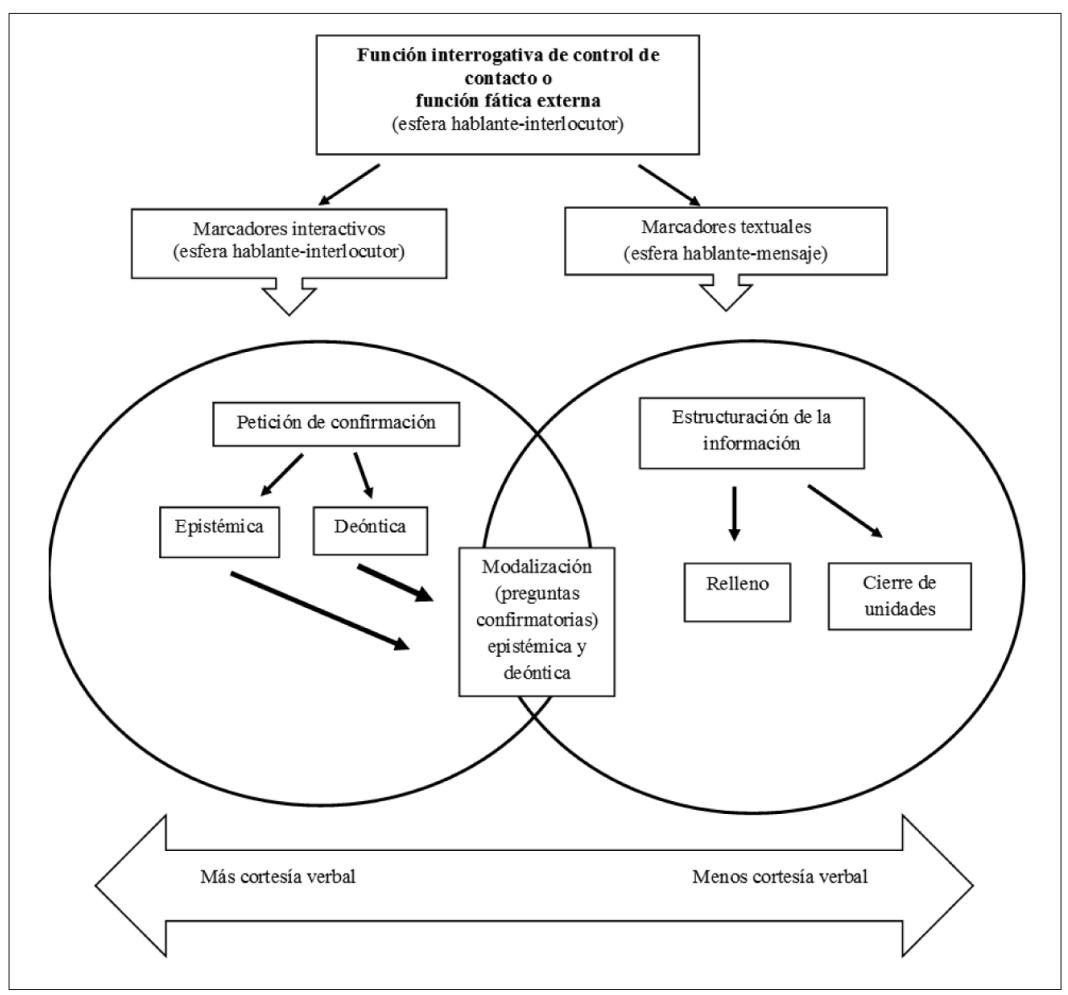

$8 \quad$ Nos referimos a un continuum porque en muchos casos es difícil determinar categóricamente la orientación de un marcador lingüístico hacia una esfera particular de la comunicación. Así, cuando proponemos valores específicos, se trata de casos prototípicos en contraste con casos periféricos de difícil clasificación, por ejemplo, casos de marcadores que evidencian una mayor motivación semántica. 


\subsubsection{Tipo de unidad discursiva en la que operan}

Los tipos de unidades discursivas que consideramos en nuestro análisis fueron los intersegmentos ${ }^{9}$, los actos, los subactos y los enunciados. También se registraron casos en los que los marcadores interrogativos de control de contacto no constituían una parte de un enunciado, sino que formaban enunciados por sí mismos, con relativa autonomía.

\subsubsection{Intersegmentos}

Los marcadores interrogativos de control de contacto pueden aparecer en medio de segmentos incompletos sintáctica e informativamente. Su función preponderante es estructuradora, específicamente de relleno, ayudando al hablante a ganar tiempo para planificar su discurso. Los marcadores que desempeñan esta función de modo preferente son ¿no cierto? y icierto?:

1) I: porque después/ es ponerle un/ un apellido al amigo/ buen amigo/ o mal amigo ya/ yo creo que no existe ni buen amigo/ ni mal amigo/ yo creo que existe el amigo [...] ahora uno puede tener ee conocer muchas personas/ tener ee ino cierto? un compañerismo/ que no es lo mismo que decir ee/ser amigo/ que tener compañerismo/ uno puede tener compañerismo con mucha gente/ puede tener afinidad con mucha gente/ tú puedes estar rodeada de/ ¿no cierto? de/ entre comillas amigos/ pero si tú empiezas a estrechar el/ el círculo y de ver cuáles son las personas que contigo/ son los amigos [... $]^{10}(\mathrm{M} \mathrm{III} \mathrm{H130})^{11}$.

Así llamaremos, de manera provisoria, a esta suerte de pseudosegmentos. Cortés y Camacho (2005) se refieren a ellos como unidades fragmentarias que no pueden ser consideradas unidades discursivas y que pueden producirse debido a problemas de planificación. Pese a los inconvenientes en su delimitación, este término provisorio alude a la función de los marcadores interrogativos de control de contacto cuando están insertos en segmentos sintáctica e informativamente incompletos o fragmentarios (Cortés y Camacho, 2005, p. 129).

10 Respecto de la transcripción de los ejemplos, creemos necesario indicar las siguientes convenciones gráficas: 1) cuando corresponda a ejemplos de mayor extensión, E = entrevistador e I = informante; 2) como se trata de variables no fonéticas y, por lo tanto, la pronunciación no es el foco de nuestro análisis, el texto correspondiente a cada ejemplo se transcribe en ortografía convencional, incluidos los acentos gráficos; 3) con el propósito de no desnaturalizar demasiado la representación del habla chilena, se ha conservado la manifestación gráfica de determinados usos característicos del español hablado en Chile, como el uso del voseo paradigmático con elisión de -s, por ejemplo, estái, soi, viví (estáis, sois, vivís con valor de segunda persona del singular, tú) y el empleo de marcadores del discurso, como po (pues); 4) para la mejor comprensión de los ejemplos incluidos en este estudio, se ha suprimido el sistema de etiquetas pertenecientes al Standard Generalized Markup Language (SGML) contenidas en el Text Encoding Initiative (TEI), que se emplea en la transcripción de las entrevistas de ESECH, excepto las pausas que se señalan con / 5) destacamos en cursiva los marcadores interrogativos de control de contacto pertinentes para cada ejemplo.

11 Al final de cada ejemplo, se indica, entre paréntesis, el código del informante, según las siguientes convenciones: grupo socioeconómico ( $\mathrm{MA}=$ medio alto, $\mathrm{M}=$ medio, $\mathrm{MB}=$ medio bajo, $\mathrm{B}=$ bajo), grupo etario (III = adultos mayores de 55 años y más, II = sujetos adultos de edad intermedia entre 35 y 54 años 
2) I: [...] entonces/ eso me quedó muy grabado/ eso quiere decir/ ¿no cierto?/ de que/ es muy probable de que el número de la disposición en el caso nuestro no sea tan importante si tú sabes el concepto/ y además porque ella/ se metía en lo de ella/ y le importaba un comino ¿no cierto?/ el resto/ (MA III M188).

Estos marcadores podrían ser sustituidos por un marcador de relleno prototípico en el español como eh (Rodríguez, 2009).

\subsubsection{Subactos y actos}

Junto con el enunciado, estas unidades discursivas son los segmentos prototípicos en que van insertos los marcadores interrogativos de control de contacto, los que se posicionan al margen. Por esta razón se los llama comúnmente "apéndices", aunque no se trata del término más adecuado, debido a que también pueden hallarse en otras posiciones (Rodríguez, 2009). En subactos y actos los marcadores interrogativos cumplen su función prototípica o nuclear de control de contacto o fática externa y son susceptibles de ser conmutados por una pregunta como " $i$ entiendes lo que digo?". Aunque no es el único, el marcador más utilizado para cumplir esta función es ¿cachái?:

3) I.: aa/ sí carreteo con vos po huevón ¿cachái?/ carreteo/ puta/ salgo con puras personas universitarias/ igual/ igual tengo mi/ mi grupo así como/ el/ el/ el carrete flaite ¿cachái?/ como/ vamos a puro tomar/ y puro/ a puro poncear// pero/ me gusta más estar con ustedes que con los otros/ porque/ puta/ tenimos horarios losotros para carretear/ como el lobby / tomi/ tomar/ ¿cachái?/ conversar/ tirar la talla/ aprender un poquito más del mundo/ y y/ yo aprendo harto igual/¿cachái?/ y hasta que es momento que tenimos que bailar/ "pongámonos a bailar/ hueveamos" "ya/ hueveamos"/ llegó la mañana/ y seguimos hueveando ¿cachái?/ almorz/ tomamos desayuno huevón/ los vinimos a la casa tuya/ yy/ yy/ hasta el almuerzo po huevón/ ¿cachái?/ esos son mis amigos (B I H005).

4) I: en este momento me gustaría sí estar con buena salud/ es que si no estái sonada E: claro/I: de verdad/ y y físicamente en condiciones/ o sea que tú físicamente te puedas desplazar y mover bien/ ¿te fijah?/

y I = hablantes jóvenes de entre 20 y 34 años) y sexo ( $\mathrm{M}=$ mujer y $\mathrm{H}$ = hombre $)$. A continuación del sexo se señala el número correlativo del sujeto en el corpus. 
porque/ ee la falta de energía sobre todo en mi trabajo si yo voy a construcciones y cosas o sea/ por eso por eso además yo hago ejercicio ite fijah? para mantenerte bien/ (MA III M185).

Los marcadores en estos ejemplos no modalizan, sino que intentan más bien "cautelar la recepción e interpretación eficiente de su mensaje por parte del interlocutor" (San Martín, 2011, p. 139). A menudo, estos marcadores se emplean con una alta frecuencia, incluso dentro del mismo enunciado (ver 3), por lo que se ha propuesto que poseen un carácter meramente expletivo o innecesario, conformando verdaderos "tics lingüísticos" (Rabanales y Contreras, 1990). Sin embargo, Briz (2001) y Portolés (2001) destacan que no se trata de elementos innecesarios, pues "poseen un valor para el buen funcionamiento de la conversación" (Portolés, 2001, p. 133-134). En este sentido, el uso recurrente de ¿cachái? puede interpretarse como un intento de mostrarse colaborativo con el interlocutor, mediante el refuerzo del contacto y el control de la comprensión de su mensaje (San Martín, 2011, p. 155).

También estos marcadores pueden desempeñar las funciones de petición de confirmación epistémica o deóntica de la información en este tipo de segmento:

5) I: a mí me gusta su rapidez/ me gusta ee/ la transparencia que se produce/ ee pero de repente se hace mucho show del asunto iah?/ ee pero yo creo que/ de manera/bien llevado/ yo creo que eso se va a perfeccionar cada día/ ¿te fijah?/ ee yo que nací en el otro sistema del derecho/ te puedo decir que/ yo tuve juicios que duraron catorce años por ejemplo/(MA III M188).

6) E: ni tiempo tampoco I: yo diría que el tiempo uno se lo puede hacer ¿ah?/yo diría que en el fondo uno es así un poco dejado/ pero uno se puede hacer un tiempo para ir a un gimnasio/ aunque sea en la tarde un rato un par de veces a la semana/ pero a veces uno se pone más dejado no no no lo hace y debería hacerlo/ (MA III H181).

En estos ejemplos, estos marcadores podrían sustituirse por una pregunta como “ ¿no crees lo mismo?” o “ ¿no te parece a ti?”. Igualmente, aunque con menor frecuencia, notamos casos de modalización propiamente tal, es decir, en los que efectivamente se refuerza o atenúa una opinión o aseveración como en:

7) I: y se ven muy muy mal// se ven muy pésimo hablando así/ o sea no/ cero feminidad/ o sea cero cero/ y después dicen "no hay hombres/ 
los hombres no me pescan" pero es que uno al final con qué se queda/ se queda con/ se queda con una mujer digamos íntegra/ no se queda con una parte no más/ y la mujer es chora que aquí que allá que "estoy fumando" que/ o sea no/ o sea uno sale en un grupo está bien/ ellas toman fuman etcétera etcétera/ pero al final uno como hombre empieza como a estudiarlo ¿cachái?/ "y esta galla qué saco yo con seguir con ella/ mira es re buena para el trago/ es re buena para esto para esto otro para el cigarro/ o sea no/ si quiere formalizar yo creo que con esta galla no me quedo” de todas maneras ¿te fijái?/ (MA III H181).

En este ejemplo, la sustitución por una pregunta como ¿no crees lo mismo? no dejaría intacta la información pragmática, puesto que restaría el refuerzo enfático del enunciado.

\subsubsection{Enunciados}

Como apéndices de enunciados, los marcadores interrogativos de control de contacto pueden desempeñar todas las funciones antes vistas, excepto la de relleno. En general, notamos que frecuentemente se da la función de petición de confirmación epistémica y deóntica, con respuesta requerida, es decir, parecen estar más motivados semánticamente:

8) E: en general te gusta/ ¿no cierto? I: a mí me encanta// me encanta E: aparte que es bonito I: mm (MA II M169).

\subsubsection{Enunciados por sí mismos}

Por último, este tipo de marcadores pueden no estar anclados a un enunciado, sino que conformar una unidad discursiva, constituyendo así un enunciado por sí mismo, que no puede entenderse desde un punto de vista monológico, pues siempre conforma un turno reactivo, es decir, una respuesta al turno previo. Notamos aquí una predominancia de la cortesía verbal, puesto que el hablante pregunta en relación con la información proposicional o pragmática dada previamente en el turno anterior del interlocutor. Pese a que el hablante tiene claridad sobre el contenido del enunciado, solicita corroborar información evidente o implícitamente dada por el interlocutor, violando, en consecuencia, la máxima de relevancia a favor del principio de cortesía (Vigara Tauste, 1992, p. 391; Landone, 2009, p. 242):

9) E: ¿cuál es la que prefieres? ¿convivir o pololear? I: pololear lejos E: ¿de verdad? I: sí sí porque convivir ya es como/ ya no sé po empiezan 
los problemas cuando hay problemas económicos tení problemas con tu pareja ¿cachái? porque aunque suene feo ¿cachái? ee la plata es como igual en parte la felicidad de una pareja/ entoncesigual (B I M010).

\subsubsection{Posición preferente en la unidad discursiva}

Las posiciones discursivas que consideramos en nuestro análisis fueron la inicial, la intermedia y la final. La posición más frecuente en la que se encuentran los marcadores interrogativos de control de contacto es la final de unidad discursiva, ya sea subacto, acto o enunciado.

\subsubsection{Inicial}

$\mathrm{Al}$ igual que en el caso de que constituyan un turno por sí mismos, los marcadores interrogativos de control de contacto que se encuentran en esta posición suelen tener el valor de petición de confirmación deóntica o epistémica, con una fuerte presencia de cortesía verbal. Los enunciados en los que van incluidos son siempre intervenciones reactivas, por lo que deben ser entendidos dentro de un marco dialógico. Estas intervenciones son, en efecto, una respuesta al turno anterior. En estricto sentido, funcionan como una manera de corroborar la información pragmática o proposicional que el interlocutor acaba de proporcionar:

10)|E: ¿cuáles son algunos de los rasgos que caracterizan a las personas de clase alta de Santiago? los cuicos I: su manera de hablar E: ¿sí? ¿cómo hablan? (B I M010).

\subsubsection{Intermedia}

Como vimos anteriormente, los marcadores interrogativos de control de contacto solo ocurren en posición intermedia cuando se hallan entre actos y subactos. La función prototípica en esta posición es la de relleno:

11)I: me encanta el campo también $¿ a h$ ?/ es que me gusta la naturaleza/ todo lo que sea naturaleza/ yo siempre me ando fijando en la naturaleza/ en los pajaritos// me encanta// si me dices pucha "te convido al campo" pucha/ voy feliz también al campo// cuando chica pasaba mucho en el campo// pero me encanta mirar el mar/ las olas/ como que no me aburro/ es como mirar una llama/ ¿te fijái?/ de de fuego/ que tú le vai viendo como formas/ lo mismo me pasa con el mar//(M III M140). 


\subsubsection{Final}

Se trata de la posición prototípica de este tipo de marcadores. Una de las funciones secundarias que desempeñan enesta posición es la de marcador de cierre. Junto a la de relleno, consiste en la segunda función secundaria que encontramos para los marcadores interrogativos de control de contacto concerniente a la esfera de la estructuración del discurso o macrofunción textual (Cortés y Camacho, 2005, p. 144). En los siguientes ejemplos, el marcador puede ir, de manera complementaria a las funciones nucleares de carácter más fático, al término de unidades discursivas, sobre todo cuando se emplea de manera recurrente:

12)E: ya/ ¿y si te ganárai el premio mayor/ qué haríai con toda la plata?// I: lo primero que haría/ me aseguraría así/ aseguraría a mi familia ¿cachái?/ me compraría una casa ¿cachái?/ impecable/ su auto ¿cachái?/todo/moto/ asegurarte y/ ee/ poner un negocio po / ¿cachái?/ un negocio que te genere la plata ¿cachái?/ y vai ahí/ aumentando o manteniéndolo ¿cachái?/ para que no la desaprovechí po/ si a las finales una oportunidad así no la vai a tener dos veces así// y un negocio que te mantenga el nivel que vai a querer vivir/ ¿cachái?/ eso (MB I H049).

13)E: eso le iba a preguntar o sea ¿cómo puede ee el calentamiento global estar influenciando en esto del clima?// I: esa/ en ese sentido/ en ese sentido de que nosotros estamos contaminando demasiado la atmósfera/ se están produciendo estos hoyos estos hoyos negros ¿no eh cierto?/ estamos quemando en el fondo la atmósfera/ estamos permitiendo que queque en el fondo la capa que nos protege de a poco se vaya debili debilitando ¿no eh cierto?/ y en algunas partes ya sencillamente se debilitó completamente/ y permite que pasen/ además radiación solar más/ mayor cantidad de de rayos rayos ee nocivos para la piel para la salud etcétera/ es por el efecto por por el efecto de nosotros mismos digamos/ por el hecho de que estamos tirando mucho/ mucha contaminación a la al aire/ de todo tipo// (MA III H181).

Todos estos ejemplos tienen en común que los marcadores interrogativos están posicionados en segmentos no necesariamente relevantes en relación con otros, en particular, al cierre de ideas breves. De este modo, se puede interpretar que, cuando van hacia el final del enunciado, estos marcadores pueden tener un efecto de sentido de cambio de turno (ver el último caso de ¿cachái? en el ejemplo 12). 


\section{2. Análisis sociolingüístico}

Según lo que señalamos previamente, para el análisis sociolingüístico de los marcadores interrogativos de control de contacto, solo consideraremos aquellos que se emplearon más de 25 veces en la muestra, a saber, ¿̇cachái? (1499/68,8\%), ¿ya? (127/5,8\%), ¿ah? (104/4,8\%), ¿te fijah? (84/3,9\%), ¿no cierto? (71/3,3\%), ¿icierto? (66/3,0\%), ¿̇te fijái? (62/2,8\%), ¿me entendih? (53/2,4\%), ino? (47/2,2\%), ino eh cierto? (38/1,7\%) y imm? $(27 / 1,2 \%)(\mathrm{N}=2178 / 100 \%)$. Como cabría esperar, el empleo de estos marcadores se distribuye de manera bastante irregular en la muestra. De este modo, mientras hay sujetos que prácticamente no los emplean, otros lo hacen asiduamente; asimismo, mientras algunos sujetos cuentan con una amplia variedad de marcadores para esta función, otros emplean preferentemente solo uno.

\subsubsection{Sexo-género}

La tabla 2 expone la frecuencia del empleo de cada una de estas partículas, de acuerdo con el sexo-género de los hablantes:

Tabla 2. Frecuencia absoluta y porcentaje de los marcadores interrogativos de control de contacto según el sexo-género de los sujetos.

\begin{tabular}{|l|c|c|}
\hline Marcador & Hombres & Mujeres \\
\hline ¿cachái? & $951(63,4 \%)$ & $548(36,6 \%)$ \\
\hline ¿ya? & $94(74,0 \%)$ & $33(26,0 \%)$ \\
\hline ¿ah? & $60(57,7 \%)$ & $44(42,3 \%)$ \\
\hline ¿te fijah? & $31(36,9 \%)$ & $53(63,1 \%)$ \\
\hline ¿no cierto? & $43(60,6 \%)$ & $28(39,4 \%)$ \\
\hline ¿cierto? & $40(60,6 \%)$ & $26(39,4 \%)$ \\
\hline ¿te fijái? & $15(24,2 \%)$ & $47(75,8 \%)$ \\
\hline ¿me entendíh? & $3(5,7 \%)$ & $50(94,3 \%)$ \\
\hline ¿no? & $36(76,6 \%)$ & $11(23,4 \%)$ \\
\hline ¿no eh cierto? & $33(86,8 \%)$ & $5(13,2 \%)$ \\
\hline ¿mm? & $26(96,3 \%)$ & $1(3,7 \%)$ \\
\hline Total & $1332(61,2 \%)$ & $846(38,8 \%)$ \\
\hline
\end{tabular}

Como observamos, en nuestro corpus, en términos estadísticos descriptivos, los hombres utilizaron más este tipo de marcadores que las mujeres. Desde un punto de vista estadístico inferencial, la media de los 
hombres fue 22, 665 y la de las mujeres 15, 283. Con todo, los resultados de ANOVA fueron $\mathrm{F}=1,444 \mathrm{y} \mathrm{p}=0,232 \mathrm{y}$ los de Kruskal-Wallis, Chi cuadrado= 0,300 y $p=0,584$, por lo que las diferencias en el uso de los marcadores interrogativos de control de contacto entre hombres y mujeres no son significativas. En cuanto a ¿cachái?, también verificamos descriptivamente un empleo preferente en hombres, concordando con lo señalado por San Martín (2011), para quien se puede considerar a este marcador como un rasgo con prestigio encubierto (Labov, 1983; Trudgill y Hernández Campoy, 2007, p. 254), más específicamente, como un "indicador de vernacularidad" (San Martín, 2011, p. 161). No obstante, la aplicación de las pruebas ANOVA $(\mathrm{F}=1,359 ; \mathrm{p}=0,246)$ y Kruskal-Wallis (Chi cuadrado= 0,$000 ; \mathrm{p}=0,984)$ no avala esta asociación entre ¿̇cachái? y el discurso masculino.

\subsubsection{Edad}

La tabla 3 presenta la frecuencia del empleo de los marcadores seleccionados, de acuerdo con la edad de los hablantes:

Tabla 3. Frecuencia absoluta y porcentaje de los marcadores interrogativos de control de contacto según edad de los sujetos.

\begin{tabular}{|l|c|c|c|}
\hline Marcador & $\mathbf{2 0 - 3 4}$ & $\mathbf{3 5 - 5 4}$ & $\mathbf{5 5}$ y más \\
\hline ¿cachái? & $1271(84,8 \%)$ & $187(12,5 \%)$ & $41(2,7 \%)$ \\
\hline ¿ya? & $4(3,1 \%)$ & $13(10,2 \%)$ & $110(86,6 \%)$ \\
\hline ¿ah? & $3(2,9 \%)$ & $26(25,0 \%)$ & $75(72,1 \%)$ \\
\hline ¿te fijah? & $0(0,0 \%)$ & $32(38,1 \%)$ & $52(61,9 \%)$ \\
\hline ¿no cierto? & $2(2,8 \%)$ & $5(7,0 \%)$ & $64(90,1 \%)$ \\
\hline ¿cierto? & $3(4,5 \%)$ & $48(72,7 \%)$ & $15(22,7 \%)$ \\
\hline ¿te fijái? & $0(0,0 \%)$ & $34(54,8 \%)$ & $28(45,2 \%)$ \\
\hline ¿me entendíh? & $7(13,2 \%)$ & $24(45,3 \%)$ & $22(41,5 \%)$ \\
\hline ¿no? & $3(6,4 \%)$ & $7(14,9 \%)$ & $37(78,7 \%)$ \\
\hline ¿no eh cierto? & $0(0,0 \%)$ & $0(0,0 \%)$ & $38(100,0 \%)$ \\
\hline ¿mm? & $0(0,0 \%)$ & $0(0,0 \%)$ & $27(100,0 \%)$ \\
\hline Total & $1293(59,4 \%)$ & $376(17,3 \%)$ & $509(23,45 \%)$ \\
\hline
\end{tabular}

Como se ve, porcentualmente, casi el $60 \%$ de los casos registrados se concentra en el grupo más joven de la muestra. Asimismo, se aprecia una mayor variedad en el empleo de marcadores interrogativos de control de contacto en los grupos etarios mayores que en el grupo 
juvenil que se inclina hacia ¿cachái?, casi de modo exclusivo. De este modo, la variable edad fue la más sensible al empleo de los marcadores interrogativos de control de contacto, como destaca San Martín (2011, p. 159). Los resultados de ANOVA $(\mathrm{F}=5,682 ; \mathrm{p}=0,004)$ y de KruskalWallis (Chi cuadrado $=9,229 ; p=0,010$ ) comprueban la significación estadística de este factor. Las medias correspondientes son 33,350 (2034); 9,950 (35-54) y 14, 500 (55 y más años), evidenciando un patrón abrupto en el comportamiento de este tipo de marcadores en relación con la variable etaria (ver gráfico 1). Por lo tanto, verificamos una mayor predisposición en el grupo juvenil hacia el control de contacto, esto es, hacia el requerimiento de confirmarla comprensión de su discurso por parte de su interlocutor.

Gráfico 1. Medias marginales estimadas de los marcadores interrogativos de control de contacto según la edad de los sujetos.

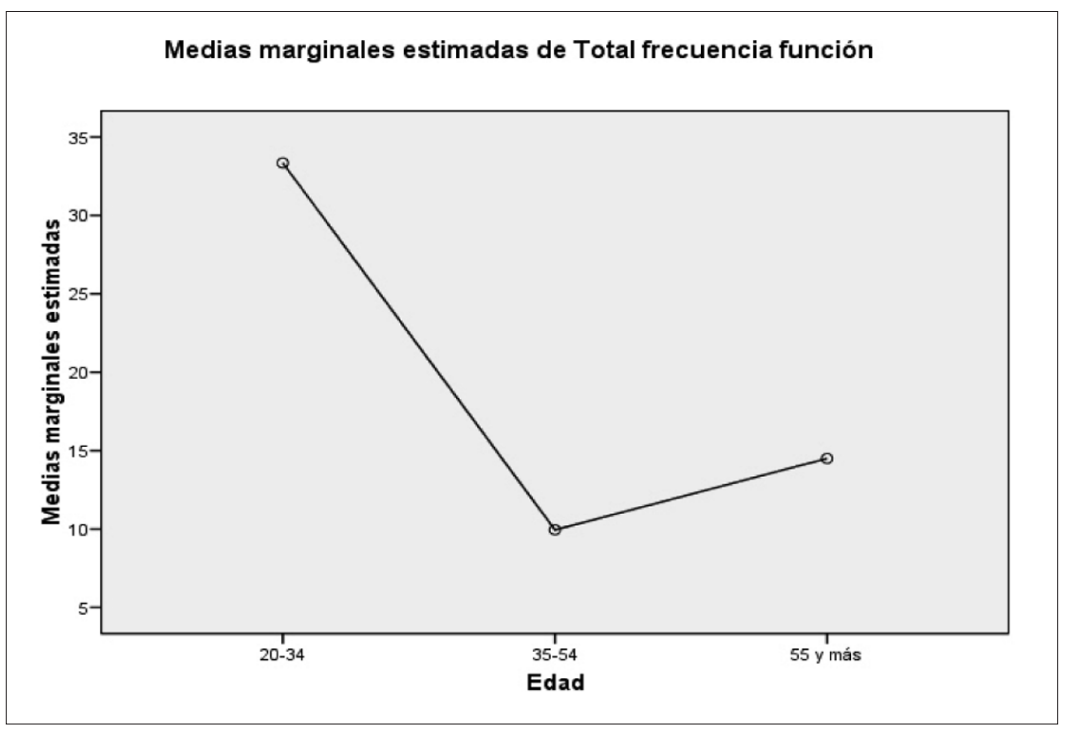

Por lo que refiere al comportamiento de marcadores interrogativos de control de contacto específicos, destacamos los siguientes casos:

a) El contraste de medias de ¿cachái? arroja los siguientes valores: 31,775 (20-34), 4,675 (35-54) y 1,025 (55 y más años), según un patrón también abrupto (ver gráfico 2). La prueba paramétrica $(\mathrm{F}=13,715 ; \mathrm{p}=0,000)$ y la no paramétrica (Chi cuadrado $=44,267 ; \mathrm{p}=0,000$ ) confirman la significatividad estadística de esta asociación entre ¿cachái? y los hablantes más jóvenes (cf. San Martín, 2011, p. 159). 
Gráfico 2. Medias marginales estimadas del marcador interrogativo de control de contacto ¿cachái? según la edad de los sujetos.

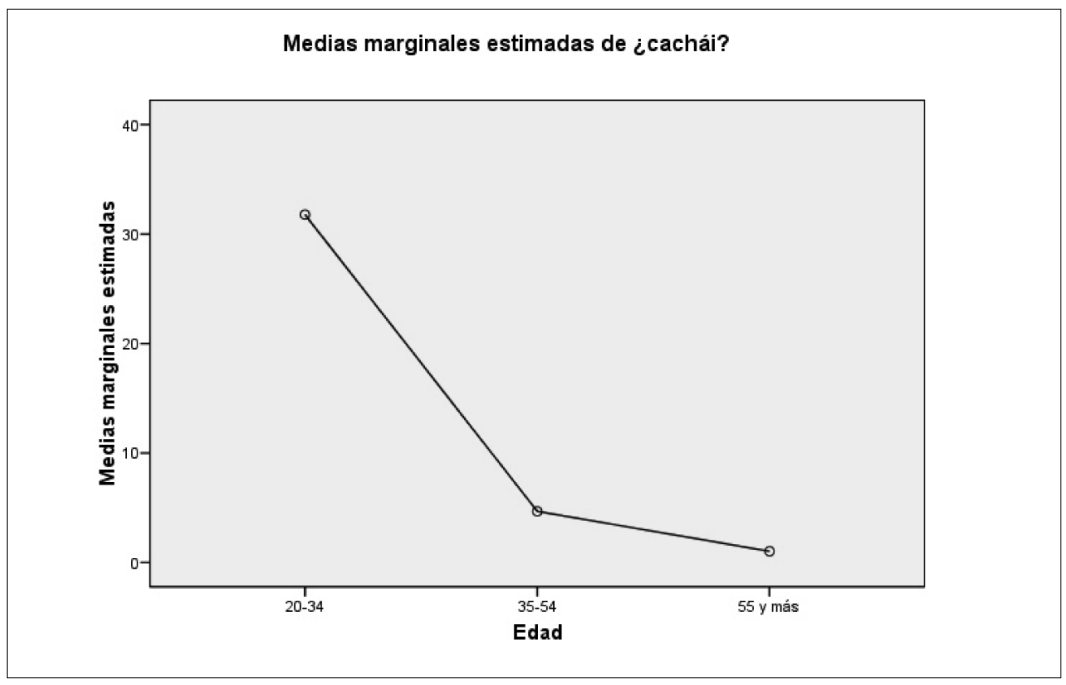

b) La varianza de $i y a$ ? indica las siguientes medias: 0,100 (20-34), 0,035 (35-54) y 2,750 (55 y másaños), representando también un patrón abrupto (ver gráfico 3). Tanto ANOVA $(\mathrm{F}=4,899 ; \mathrm{p}=0,009)$ como Kruskal-Wallis $(10,851 ; p=0,004)$ avalan la significación estadística de la asociación entre ¿ya? y los hablantes de mayor edad.

Gráfico 3. Medias marginales estimadas del marcador interrogativo de control de contacto ¿ya? según la edad de los sujetos.

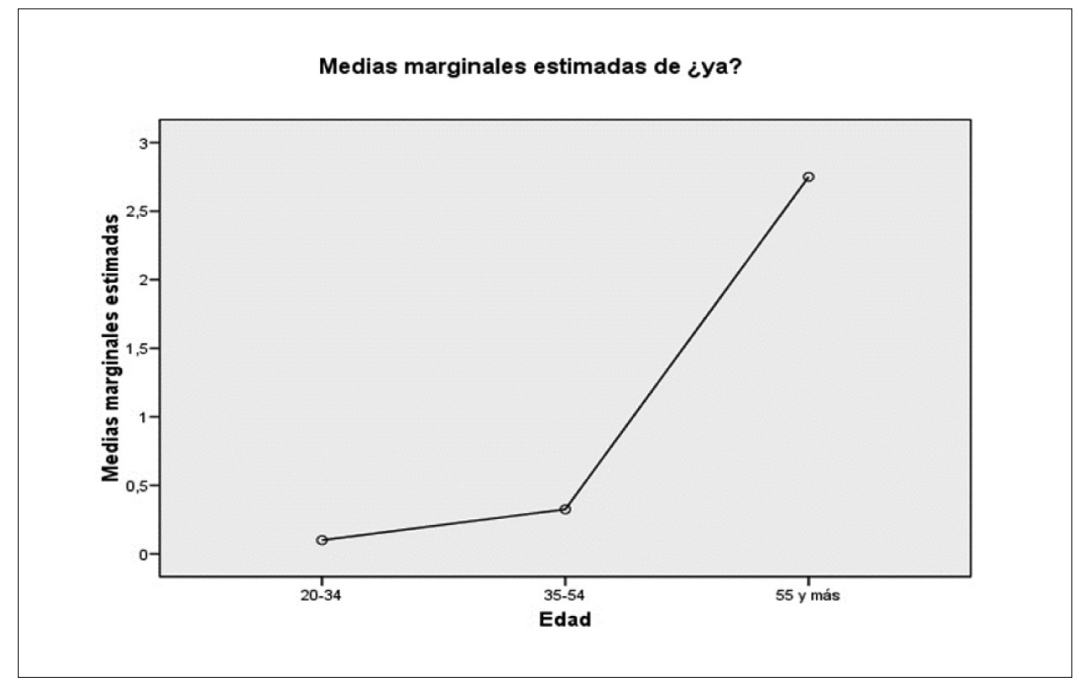


En consecuencia, ¿cachái? y ¿ya? se comportan de modo inverso en relación con la edad de los sujetos: el primero es característicamente juvenil mientras el segundo lo es del discurso de los hablantes mayores.

\subsubsection{Grupo socioeconómico}

La tabla 4 especifica la frecuencia del empleo de los marcadores considerados, de acuerdo con el grupo socioeconómico de los hablantes:

Tabla 4. Frecuencia absoluta y porcentaje de los marcadores interrogativos de control de contacto según el grupo socioeconómico de los sujetos.

\begin{tabular}{|l|c|c|c|c|}
\hline Marcador & Bajo & Medio Bajo & Medio & Medio Alto \\
\hline ¿cachái? & $367(24,5 \%)$ & $546(36,4 \%)$ & $182(12,1 \%)$ & $404(27,0 \%)$ \\
\hline ¿ya? & $3(2,4 \%)$ & $4(3,1 \%)$ & $77(60,6 \%)$ & $43(33,9 \%)$ \\
\hline ¿ah? & $3(2,9 \%)$ & $44(42,3 \%)$ & $19(18,3 \%)$ & $38(36,5 \%)$ \\
\hline ¿te fijah? & $0(0,0 \%)$ & $4(4,8 \%)$ & $39(46,4 \%)$ & $41(48,8 \%)$ \\
\hline ¿no cierto? & $12(16,9 \%)$ & $5(7,0 \%)$ & $26(36,6 \%)$ & $28(39,4 \%)$ \\
\hline ¿cierto? & $8(12,1 \%)$ & $10(15,2 \%)$ & $20(30,3 \%)$ & $28(42,4 \%)$ \\
\hline ¿te fijái? & $0(0,0 \%)$ & $0(0,0 \%)$ & $45(72,6 \%)$ & $17(27,4 \%)$ \\
\hline ¿me entendíh? & $0(0,0 \%)$ & $1(1,9 \%)$ & $36(67,9 \%)$ & $16(30,2 \%)$ \\
\hline ¿no? & $3(6,4 \%)$ & $23(48,9 \%)$ & $5(10,6 \%)$ & $16(34,0 \%)$ \\
\hline ¿no eh cierto? & $0(0,0 \%)$ & $0(0,0 \%)$ & $6(15,8 \%)$ & $32(84,2 \%)$ \\
\hline ¿mm? & $1(3,7 \%)$ & $12(44,4 \%)$ & $2(7,4 \%)$ & $12(44,4 \%)$ \\
\hline Total & $397(18,2 \%)$ & $649(29,8 \%)$ & $457(21,0 \%)$ & $675(31,0 \%)$ \\
\hline
\end{tabular}

No existen diferencias notables en el empleo de los marcadores interrogativos de control de contacto, según el grupo socioeconómico de los sujetos, en términos descriptivos, aunque se verifica un patrón con forma de "s" con un uso más frecuente en los grupos medio alto y medio bajo de la escala. El contraste de las medias es el siguiente: 14,033 (Bajo); 20,739 (Medio bajo); 17,192 (Medio) y 23,933 (Medio alto). Si bien la prueba no paramétrica dio un resultado significativo (Chi cuadrado $=11,859 ; \mathrm{p}=0,008$ ), la aplicación de la prueba paramétrica $(\mathrm{F}=0,490 ; \mathrm{p}=0,690)$ no verifica dicha significación. Por consiguiente, a nuestro juicio, la asociación entre el empleo de los marcadores interrogativos de control de contacto y el grupo socioeconómico de los sujetos se trata de una posibilidad que habría que corroborar ampliando la muestra bajo análisis. Consecuentemente, el marcador del repertorio más frecuente, ¿cachái?, se distribuye de manera bastante homogénea, 
aunque también reproduce un patrón con forma de "s". La distribución de otros marcadores se concentró en los grupos medio y medio alto (por ejemplo, ¿me entendíh?, ¿ya?, ¿te fijah? y ¿te fijái?). Notamos, además, que los grupos medio y medio alto contaron con una mayor variedad de marcadores que el resto de los grupos de la muestra. Probablemente, este hecho pueda atribuirse a las diferencias de repertorio verbal entre los diferentes sociolectos considerados en nuestro análisis.

\subsubsection{Intersección entre variables}

La única intersección que dio un resultado significativo fue el cruce entre edad y sexo-género de los sujetos con respecto al empleo de ¿cachái? (ANOVA: $\mathrm{F}=3,428 ; \mathrm{p}=0,036$ ). La varianza es la siguiente: $\mathrm{I}$ ) 20-34: Hombre (43,583), Mujer (18,150); II) 35-54: Hombre (1,450), Mujer (7,900); III) 55 y más años: Hombre (0,700), Mujer (1,350) (ver gráfico 4).

Gráfico 4. Medias marginales de ¿cachái? según la edad y el sexo-género de los sujetos.

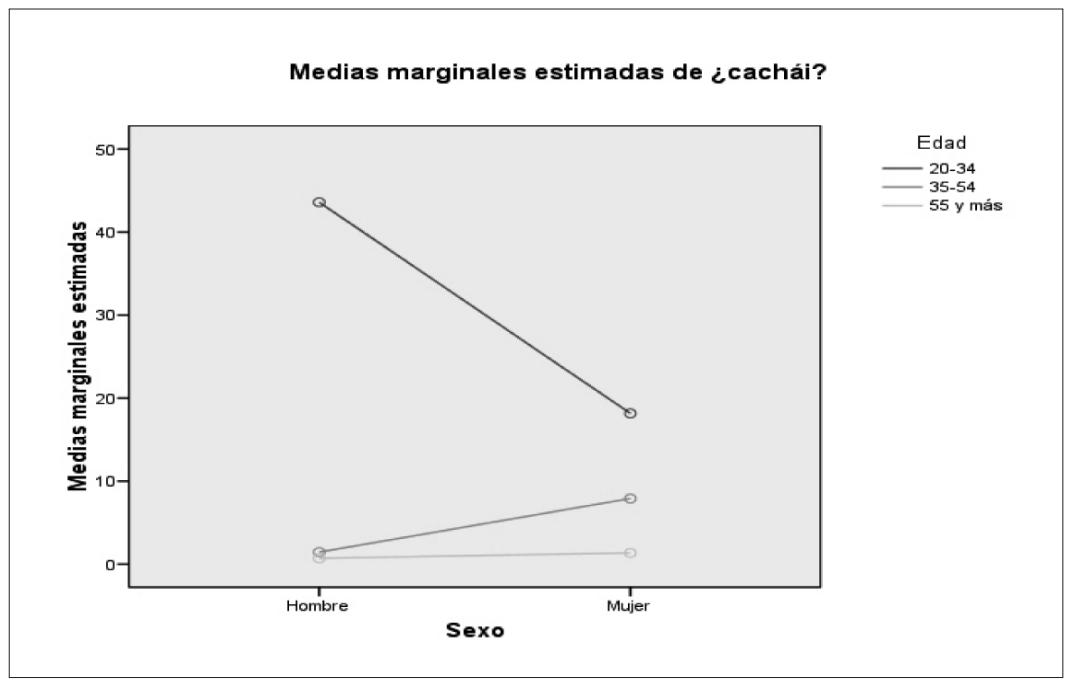

Los hombres del grupo de 20-34 años usan mucho más ¿cachái? que las mujeres del mismo grupo etario. En el grupo de 35-54 años la brecha disminuye y, en cambio, son ellas quienes tienden a emplear más ¿cachái? Finalmente, en el grupo de 55 y más años, las diferencias son irrelevantes. 


\section{Conclusiones}

Desde un punto de vista pragmático, los marcadores interrogativos de control de contacto, al igual que muchas otras partículas son polifuncionales. Sin embargo, su función nuclear es la de control de contacto o fática externa, en la que operan predominantemente en la esfera de la comunicación relacionada con el hablante y el oyente. A partir de esta función nuclear, se derivan las subfunciones de petición de confirmación deóntica y petición de confirmación epistémica. En el caso de hallarse en segmentos discursivos asertivos en que el hablante restringe las posibilidades de negociación con su interlocutor respecto del propio compromiso con su postura o el grado de certeza de su información, pueden interpretarse como modalizadores propiamente tales, configurando así "preguntas confirmatorias". En relación con el tipo de unidad en que se insertan y la posición que ocupan, cuando van en posición intermedia, dentro de los "intersegmentos", la función principal de los marcadores interrogativos de control de contacto parece ser la de relleno. En cambio, en el caso de la posición inicial de enunciado, pueden interpretarse como una reacción a un turno previo, por lo tanto, parecen operar bajo la función de petición de información deóntica y epistémica. En los usos que registramos, los marcadores interrogativos de control de contacto nunca ocuparon una posición inicial absoluta. Con respecto a su posición prototípica, esto es, la final de unidad discursiva, notamos que en actos y sub-actos pueden cumplir todas las funciones que verificamos, al igual que en enunciados, aunque a veces, al funcionar como marcadores de cierre, pueden tener un efecto de sentido de cesión de un turno de habla. De este modo, corroboramos lo señalado por Briz y Pons (2010, p. 329): "la variación funcional de los marcadores del discurso está limitada por su posición discursiva y por el tipo de unidad en que se integran [...] la unidad y la posición están en correlación en gran medida con la función de los marcadores discursivos".

Desde un punto de vista sociolingüístico, la variable etaria resultó ser la más sensible al empleo de los marcadores interrogativos de control de contacto. De este modo, los jóvenes utilizan una mayor cantidad de estos marcadores que el resto de los grupos etarios, formando un patrón abrupto. La incidencia del factor etario es evidente en el empleo de ¿cachái?, que es el marcador predominante para desempeñar la función interrogativa de control contacto y que es, también, más frecuente entre los sujetos más jóvenes; hecho que podría interpretarse como un 
cambio lingüístico en marcha (San Martín, 2011, p. 162). Asimismo, la edad de los sujetos es determinante en el empleo del marcador $i y a$ ?, aunque de modo inverso, puesto que su uso es mayoritario en sujetos de mayor edad, si bien su frecuencia relativa es bastante inferior a la de $i c a c h a ́ i$ ? La única intersección de factores que dio un resultado significativo fue el cruce entre la edad y el sexo-género de los sujetos con respecto a ¿cachái?, ya que son los hombres jóvenes los que más emplean dicho marcador específico. Estos resultados nos permiten confirmar las hipótesis de base de nuestro trabajo, esto es, a) ¿cachái? es el marcador interrogativo de control de contacto más frecuente, aunque hay un repertorio variado de marcadores que desempeñan esta función; b) a pesar de cumplir una función fática nuclear, los marcadores que la cumplen presentan subvalores que los hace ser polifuncionales y c) los factores sociodemográficos de los sujetos inciden de manera irregular en su empleo, ya que el factor etario es el más sensible, estadísticamente, mientras el sexo-género solo resulta de interés en combinación con el primero con respecto al uso de ¿cachái? y el grupo socioeconómico es, en la práctica, irrelevante.

Para finalizar, es importante destacar algunas de las limitaciones y proyecciones de nuestro estudio. Por un lado, un enfoque semasiológico, distinto al onomasiológico aquí desarrollado, podría describir valores pragmáticos específicos de algunos de los marcadores registrados, con mayor detalle. Por otro, futuros trabajos que repliquen el nuestro mediante un corpus de análisis más amplio o que contrasten nuestros resultados con datos provenientes de otras variedades de español podrían resultar muy útiles, a efecto de generalizar o relativizar nuestras conclusiones. De igual modo, a nuestro juicio, sería oportuna una investigación complementaria en la que se cuantifique el empleo de las sub-funciones de los marcadores interrogativos de control de contacto, algunas más relacionadas con la colaboración que otras, con el objeto de comprobar una nueva hipótesis, matizada con los resultados obtenidos de este estudio, de que las mujeres recurren más a las funciones de control de contacto de petición de confirmación que los hombres, quienes parecen decantarse por las funciones de modalización, de relleno y de cierre de unidad discursiva. 


\section{Referencias bibliográficas}

Briz, A. (2001). El español coloquial en la conversación: esbozo de pragmagramática. Barcelona: Ariel.

Briz, A. y S. Pons. (2010). Unidades, marcadores discursivos y posición. En Ó. Loureda y E. Acín (Coords.), Los estudios sobre marcadores del discurso, hoy (pp. 327-358). Madrid: Arco Libros.

Carbonero, P. y J. Santana. (2010). Marcadores del discurso, variación dialectal y variación social. En Ó. Loureday E. Acín (Coords.), Los estudios sobre marcadores del discurso, hoy (pp. 497-521). Madrid: Arco Libros.

Casado Velarde, M. (1998). Lingüística del texto y marcadores del discurso. En M. Martín Zorraquino y E. Durán (Coords.), Marcadores discursivos: teoría y práctica (pp. 55-70). Madrid: Arco Libros.

Cestero, A. M. (2002). La función fática del lenguaje en el discurso y en la conversación. IV Congreso de Lingüística General (pp. 617-630). Universidad de Cádiz: Servicio de Publicaciones.

. (2003). La función fática del lenguaje en el discurso y en la conversación: recursos lingüísticos para llamar la atención del interlocutor. En Lengua, variación y contexto: estudios dedicados a Humberto López Morales (pp. 227-243). Madrid: Arco Libros.

Clark, H. y S. Brennan. (1991). Grounding in communication. Perspectives on socially shared cognition, (13), 127-149.

Cortés, L. y M. Camacho. (2005). Unidades de segmentación y marcadores del discurso. Madrid: Arco Libros.

Fuentes, C. (1990a). Algunos operadores de función fática. Sociolingüística Andaluza, (5), 137-150.

(199ob). Apéndices con valor apelativo. Sociolingüística Andaluza (5), 171-196.

García Vizcaíno, M. J. (2005). El uso de los apéndices modalizadores ¿no? y ieh? en español peninsular. En L. Sayahi y M. Westmoreland (Eds.), Selected Proceedings of the Second Workshop on Spanish Sociolinguistics. Cascadilla Proceedings Project Somerville, MA. Recuperado de: http://www.lingref.com/cpp/wss/2/paper1143. pdf. 
Gille, J. (2015a). On the development of Chilean Spanish discourse marker cachái. Revue Romane, 50 (1), 3-29.

(2015b). Los apéndices conversacionales en la argumentación: el caso de ¿cachái? En G. Engwall y L. Fant (Eds.), Festival romanística. Contribuciones lingüisticas (pp. 239-258). Stockholm: Stockholm University Press.

Hernández Campoy, J. M. y M. Almeida. (2005). Metodología de la investigación sociolingüística. Málaga: Editorial Comares.

Jakobson, R. (1975). Lingüística y poética. En Ensayos de lingüistica general (pp. 347-395). Barcelona: Seix Barral.

Labov, W. (1983). Modelos sociolingüísticos. Madrid: Cátedra.

Landone, E. (2009). Los marcadores del discurso y la cortesía verbal en español. Berna, Peter Lang.

Lenz, R. (1905). Diccionario etimológico de las voces derivadas de lenguas americanas (Edición dirigida por M. Ferreccio Podestá). Santiago de Chile: Editorial Universitaria.

Malinowski, B. (1984 [1923]). El problema del significado en las lenguas primitivas. En Ch. K. Ogden y I. A. Richards (Eds.), El significado del significado (pp. 310-352). Barcelona: Paidós.

Martín Zorraquino, M.y J. Portolés. (1999). Los marcadores del discurso. En I. Bosque y V. Demonte (Eds.), Gramática descriptiva de la lengua española (pp. 4051-4207). Madrid: Espasa Calpe.

Mondaca, L., A. Méndez y M. Rivadeneira. (2014). “No es muletilla, es marcador, ¿cachái?”. Análisis de la función pragmática del marcador discursivo conversacional cachái en el español de Chile. Literatura y Lingüistica, (32), 233-258.

Móccero, M. L. (2010). Las preguntas confirmatorias como indicadoras de posicionamiento intersubjetivo. Estudios filológicos, (45), 67-78.

Ortega, J. (1985). Apéndices modalizadores en español: los comprobativos. Estudios románicos dedicados al profesor Andrés Soria Ortega, vol. 1 (pp. 239-255). Granada: Universidad de Granada.

(1986). Aproximación al mecanismo de la conversación: apéndices justificativos. Verba, (13), 269-290.

Pons, S. (1998). Oye y mira o los límites de la conexión. En M. A. Martín Zorraquino y E. Montolío Durán (Eds.), Marcadores discursivos: teoría y práctica (pp. 213-228). Madrid: Arco Libros. 
Portolés, J. (2001). Marcadores del discurso. Barcelona: Ariel.

Rabanales, A. y L. Contreras. (1995). Las muletillas en el habla culta de Santiago de Chile. En Scripta Philologica in honorem Juan M. Lope Blanch, Vol. 2 (pp. 673-744). México: UNAM.

Ramírez Gelbes, S. (2003). La partícula “eh” y la Teoría de la Relevancia: Un ejemplo de contenido procedimental. Estudios Filológicos, (38), 157-177.

Real Academia Española. (2001). Diccionario de la lengua española. Madrid: Espasa Calpe.

Rodríguez Muñoz, F. (2009). Estudio sobre las funciones pragmadiscursivas de $i n o$ ? y $i e h$ ? en el español hablado. RLA. Revista de lingüística teórica y aplicada, 47(1), 83-101.

San Martín, A. (2011). Los marcadores interrogativos de control de contacto en el corpus PRESEEA de Santiago de Chile. Boletín de Filología, XLVI(2), 135-166.

. (2013). Los reformuladores de distanciamiento en el corpus PRESEEA de Santiago de Chile. Boletín de Filología, XLVIII (1), 171-199.

(2015). Variantes y equivalentes funcionales de al final: los reformuladores de recapitulación en el habla santiaguina. $R L A$. Revista de Lingüística Teórica y Aplicada, 53(2), 97-119.

(2016a). Los marcadores de reformulación en el español oral de Santiago de Chile: análisis discursivo y sociolingüístico. Oralia, (19), 283-324.

(2016b). Análisis sociolingüístico de los reformuladores de rectificación en el habla santiaguina. Literatura y Lingüística, (33), 241-264.

San Martín, A. y S. Guerrero. 2015. Estudio Sociolingüístico del Español de Chile (ESECH): recogida y estratificación del corpus de Santiago. Boletín de Filología, L(1), 221-247.

Trudgill, P. y J. M. Hernández Campoy. (2007). Diccionario de sociolingüística. Madrid: Gredos.

Vigara Tauste, A. M. (1992). Morfosintaxis del español coloquial. Madrid: Gredos. 